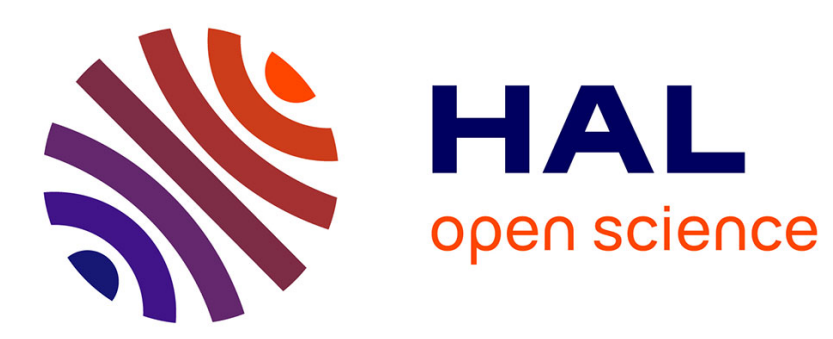

\title{
Identification et caractérisation d'un potyvirus provoquant la maladie du rabougrissement jaune du melon
}

\author{
Hervé Lecoq, Michel Pitrat, M. Clement
}

\section{- To cite this version:}

Hervé Lecoq, Michel Pitrat, M. Clement. Identification et caractérisation d'un potyvirus provoquant la maladie du rabougrissement jaune du melon. Agronomie, 1981, 1 (10), pp.827-834. 10.1051/agro:19811001 . hal-02728534

\section{HAL Id: hal-02728534 \\ https://hal.inrae.fr/hal-02728534}

Submitted on 2 Jun 2020

HAL is a multi-disciplinary open access archive for the deposit and dissemination of scientific research documents, whether they are published or not. The documents may come from teaching and research institutions in France or abroad, or from public or private research centers.
L'archive ouverte pluridisciplinaire HAL, est destinée au dépôt et à la diffusion de documents scientifiques de niveau recherche, publiés ou non, émanant des établissements d'enseignement et de recherche français ou étrangers, des laboratoires publics ou privés. 


\section{Identification et caractérisation d'un potyvirus provoquant la maladie du rabougrissement jaune du melon}

Hervé LECOQ, Michel PITRAT $\left(^{*}\right)$ \& Marcelle CLÉMENT

I.N.R.A., Station de Pathologie végétale, $\left({ }^{*}\right)$ Station d'Amélioration des Plantes maraîchères, Centre de Recherches agronomiques d'Avignon, F 84140 Montfavet.

\section{RÉSUMÉ}

Virus,

Potyvirus,

Melon,

Cucumis melo,

Cucurbitacées,

Transmission par les pucerons,

Virus du rabougrissement jaune du melon.
Le virus du rabougrissement jaune du melon (muskmelon yellow stunt virus - MYSV) a été isolé dans le Sud de la France de melons (Cucumis melo L.) présentant de graves symptômes sur le feuillage et sur les fruits. Ce virus, transmissible mécaniquement, infecte de nombreuses espèces de Cucurbitacées (dont le concombre, la courgette et la pastèque) chez lesquelles il provoque des symptômes graves ; par contre, peu d'espèces sont sensibles à l'extérieur de cette famille : 3 espèces sur 36 éprouvées ont développé des infections généralisées. 11 ne semble pas transmis par la graine chez le melon.

Le MYSV présente des particules flexueuses de $730 \mathrm{~nm}$ de long souvent associées, dans les extraits bruts, à des structures paracristallines. Il est transmis par Myzus persicae Sulz. et Aphis gossypii Glov. sur le mode non persistant.

Une étude réalisée en immunodiffusion en milieu gélosé contenant du SDS n'a pas permis d'établir de relation sérologique entre le MYSV et les virus de la mosaïque de la pastèque type 1 (WMV1) ou type « Maroc» (WMV-MOR) et le virus de la mosaique jaune du haricot (BYMV). Par contrc, le MYSV présente une légère parenté sérologique avec le virus de la mosaïque de la pastèque type 2 (WMV2). Celle-ci n'est cependant décelable qu'avec des sérums ayant des titres élevés.

Deux pathotypes de MYSV ont pu être différenciés d'après leur aptitude à induire une réaction de flétrissement chez certaines variétés de melon. Les propriétés de ces 2 pathotypes, qui sont par ailleurs identiques, permettent de rapprocher ce virus du groupe des Potyvirus.

\section{SUMMARY}

Virus,

Potyvirus,

Muskmelon,

Cucumis melo,

Cucurbits,

Aphid transmission,

Muskmelon Yellow Stunt

Virus.
Identification and characterization of a potyvirus that induces muskmelon yellow stunt.

A virus tentatively named muskmelon yellow stunt virus (MYSV) has been isolated from muskmelon (Cucumis melo L.) in southern France. Leaves from infected plants showed severe yellowing, deformations, enations and plants were drastically stunted. Isolates from southwestern France (such as isolate E15) induced mottle and hardening of the fruit flesh while southeastern isolates (such as isolate E9) were also associated with cracks of the fruits and with a wilting reaction of some muskmelon cultivars.

MYSV is easily mechanically transmitted. It is able to infect systemically many Cucurbitaceae including cucumber, summer squash and watermelon. It also infects $C$. melo var. B633 resistant to WMV1 and $L u f f a$ acutangula Roxb. immune to WMV2. MYSV has a limited host range outside the Cucurbits : it induces local lesions on 5 out of 36 species tested (but not on Lavatera trimestris L.) and infects systematically only 3 of them (and not Pisum sativum L. and Phaseolus vulgaris L. cultivars susceptible to WMV2).

Virus particles appear as long flexuous filaments with a $730 \mathrm{~nm}$ average length and are associated in crude extracts with paracrystalline inclusions. MYSV is transmitted in a non persistent manner by Aphis gossypii Glov. and Myzus persicae Sulz. These properties suggest that MYSV is a member of the Potyvirus group. MYSV does not react in SDS immunodiffusion tests with serums prepared against WMV1, WMV-MOR and BYMV, neither these viruses reacted with an antiserum prepared against MYSV. MYSV is slightly serologically related to WMV2 but this is observed only with high titered antiserums. This distant relationship and major differences in host ranges suggest that MYSV and WMV2 are separate entities.

MYSV is apparently not seed transmitted in muskmelon but it severely reduces the seed weight and the germination rate.

Two pathotypes of MYSV are defined according to their ability (pathotype F) or unability (pathotype NF) to induce a wilting reaction in C. melo var. «Doublon».

\section{INTRODUCTION}

Au cours de l'année 1979, des symptômes viraux particulièrement graves ont été observés dans de nombreuses cultures de melon (Cucumis melo L.) du Sud de la France. Ceux-ci différaient notablement des symptômes de mosaïque associés au virus de la mosaïque du concombre (CMV), très fréquent dans les melonnières françaises (MESSIAEN $e t$ 
al., 1963, QUIOT et al., 1979), ou du « vein banding " induit par le virus de la mosaïque de la pastèque type 2 (WMV2) (LuIS ARTEAGA et al., 1976). Deux faciès maladifs prédominaient :

Dans le Sud-Ouest (région de Nérac, Lot-et-Garonne), les plantes présentaient un jaunissement général, parfois accompagné de nécroses sur les tiges et de cloques vert foncé sur les feuilles. Les fruits produits par de telles plantes avaient une chair dure et marbrée (fig. 2) ce qui les rendait incomestibles : près de la moitié de la récolte a été détruite de ce fait dans cette région.

Dans le Sud-Est (région d'Avignon, Vaucluse) des symptômes de flétrissement ainsi que le développement de craquelures sur l'écorce des fruits (fig. 4) étaient en outre observés chez certaines variétés.

Dans les 2 cas, il a été possible d'isoler des plantes présentant de tels symptômes un virus flexueux souvent associé au CMV.

Nous décrivons ici les principales propriétés de ce virus flexueux que nous proposons de dénommer le virus du rabougrissement jaune du melon (Muskmelon Yellow Stunt Virus, MYSV) en raison des symptômes qu'il induit chez la plupart des variétés de melon, hôte duquel ce virus a été isolé pour la première fois.

Plusieurs virus présentant des particules flexueuses ont été décrits chez le melon:

- Certains se rattachent au groupe des Carlavirus: le Muskmelon Vein Necrosis Virus (MVNV), décrit en Californie par FREITAG \& MiLNE (1970), et le virus de la mosaïque de la bryone, présent en France (SPIRE et al., 1966 ; QuiOT et al., 1979).

- D'autres appartiennent au groupe des Potyvirus : le virus de la mosaïque de la pastèque type 1 (WMV1) dont la gamme d'hôtes est essentiellement restreinte aux Cucurbitacées, et les virus de la mosaïque de la pastèque type 2 (WMV2) et type "Maroc» (WMV-MOR) qui sont capables d'infecter de nombreuses espèces à l'extérieur de cette famille. Ces 3 virus ne sont pas reliés sérologiquement entre eux (PurCIFUll \& HieberT, 1979 ; BaUm et al., 1979) et présentent des gammes d'hôtes différentes. Ceci indique que ces virus doivent être considérés comme des entités différentes et la nécessité d'une dénomination plus claire a déjà été suggérée (Russo et al., 1979). Plus récemment le virus du mottle de la bryone (BrMV) a été décrit comme susceptible d'infecter certaines variétés de melon. Il diffère par ses propriétés antigéniques et sa gamme d'hôte des 3 virus précédents (LOCKHART \& FisCHER, 1979).

C'est avec le WMV2 que le MYSV présente le plus de propriétés et d'hôtes en commun. C'est pour le différencier de ce virus que nous avons, dans cette étude, comparé 2 isolats de MYSV et un isolat de référence de WMV2.

\section{MATÉRIEL ET MÉTHODES}

\section{A. Isolats de virus}

Trois isolats ont été utilisés : l'isolat E9 provenant de la région d'Avignon (Vaucluse), l'isolat E15 provenant de la région de Nérac (Lot-et-Garonne) et l'isolat de WMV2 décrit par LuIS ARTEAGA et al. (1976) comme étant semblable à l'isolat de WMV2 de la collection type américaine (ATCC). Cet isolat sera dénommé ici WMV2-FR.

\section{B. Matériel végétal}

Les graines sont semées en terrine puis les plantules sont repiquées en pot contenant un mélange terreau-sable stérilisé. Elles sont élevées et mises en incubation en serres «Insect proof » maintenues à $17-25^{\circ} \mathrm{C}$.

\section{Inoculations}

Les inoculations mécaniques ont été réalisées selon la méthode couramment employée au laboratoire (MARROU, 1967). Les différentes espèces utilisées comme hôtes différentiels ont été éprouvées au moins 2 fois avec 2 ou 3 plantes par répétition. Après 4 semaines d'incubation, des rétroinoculations ont été systématiquement faites, à partir de l'apex de ces plantes, à 2 melons var. «Doublon ».

Les inoculations par pucerons ont été réalisées selon la méthode décrite par LECOQ et al. (1979). Après un jeûne de 1-2 $\mathrm{h}$, une période d'acquisition d'environ $3 \mathrm{~min}$ est accordée aux pucerons sur une plante virosée ( 2 à 3 semaines après son inoculation mécanique). Ceux-ci sont ensuite déposés sur de jeunes melons à inoculer (au stade 1 feuille). Deux à 3 heures plus tard, les plantes sont traitées par fumigation avec un insecticide avant d'être mises en incubation en serre.

\section{Microscopie électronique}

Les observations ont été faites à l'aide d'un microscope électronique Philips EM 301, après coloration négative au molybdate d'ammonium (1 p. 100, pH 7). La mensuration des particules a été faite en référence à une réplique quadrillée de carbone (calibrée à $463 \mathrm{~nm}$ ).

\section{E. Purification}

L'isolat E15 a été purifié à partir de courgette (Cucurbita pepo L. var. «Diamant "), 3 à 4 semaines après leur inoculation mécanique, selon la méthode décrite par PURCIFULL \& HiEBERT (1979), pour le WMV2. Environ $150 \mathrm{~g}$ de feuilles présentant des symptômes prononcés sont homogénéisés à l'aide d'un broyeur à hélice, dans $300 \mathrm{ml}$ d'une

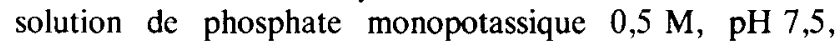
contenant 0,2 p. 100 de $\mathrm{Na}_{2} \mathrm{SO}_{4}$. Après filtration sur étamine, 8 p. 100 de n-butanol sont ajoutés et le mélange est laissé en agitation à $4{ }^{\circ} \mathrm{C}$ une nuit. Après une clarification de $10 \mathrm{~min}$ à $7500 \mathrm{tr} / \mathrm{min}$ le surnageant est filtré sur laine de verre et on lui adjoint 8 p. 100 de polyéthylène glycol (P.M. $6000 \mathrm{~d}$ ). Le mélange est laissé $1 \mathrm{~h}$ en agitation à $4{ }^{\circ} \mathrm{C}$ avant d'être soumis à une centrifugation de $15 \mathrm{~min}$ à $7500 \mathrm{tr} / \mathrm{min}$. Les culots obtenus sont repris dans une solution de phosphate monopotassique $0,025 \mathrm{M}, \mathrm{pH} 8$, contenant 0,1 p. 100 de mercaptoéthanol. La suspension est alors soumise à un double cycle de clarification $(10 \mathrm{~min}$ à $7500 \mathrm{tr} / \mathrm{min}$ ) et d'ultracentrifugation sur coussin de saccharose à 20 p. 100 (coussin de $10 \mathrm{ml}$ dans des tubes de $30 \mathrm{ml}$, $\mathrm{R} 30 \mathrm{Beckmann} 3 \mathrm{~h}$ à $29000 \mathrm{tr} / \mathrm{min}$ ). Les culots finaux sont repris dans un volume total de $1 \mathrm{ml}$ de phosphate monopotassique $0,025 \mathrm{M}, \mathrm{pH} 8$ contenant 0,1 p. 100 de mercaptoéthanol.

\section{F. Sérologie}

Un antisćrum a été obtenu vis-à-vis de l'isolat E15 après 6 injections (espacées entre elles d'une semaine) d'un mélange $1: 1$ d'une suspension de virus purifié $(5-10 \mu \mathrm{g})$ fixé par le formaldéhyde $(0,2$ p. 100$)$ et d'adjuvant incomplet de 


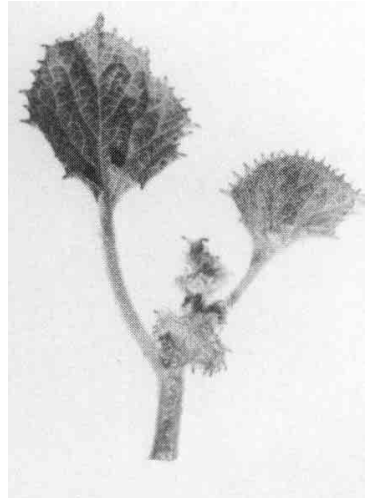

$1 a$

Figure 1a

Apex de C. melo var. "Védrantais" infecté par l'isolat E15 de MYSV montrant un éclaircissement des nervures et un arrêt de croissance (1 mois après l'inoculation).

Apex of a C. melo var. "Vedrantais" plant infected by MYSV E1S isolate showing vein clearing and stunting (1 month after inoculation).

$3 a$
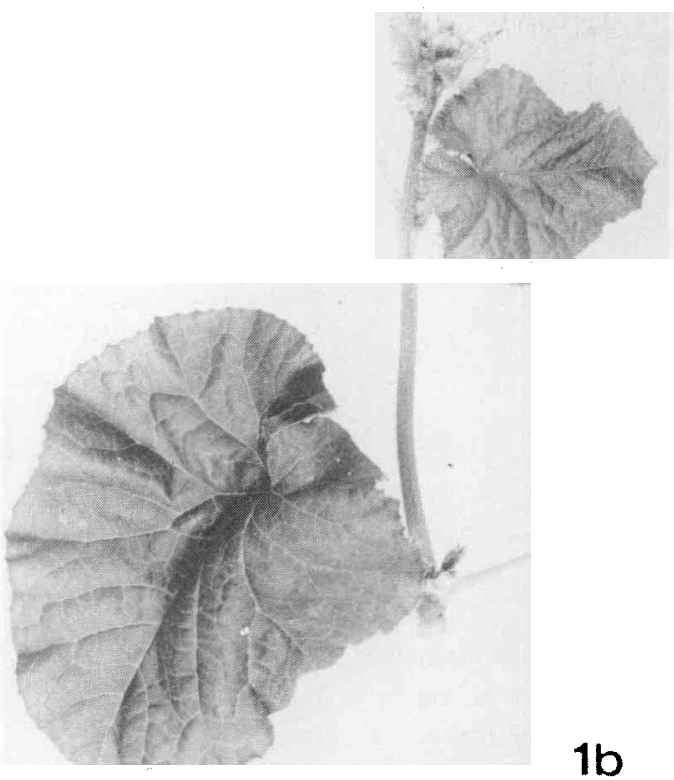

$1 b$

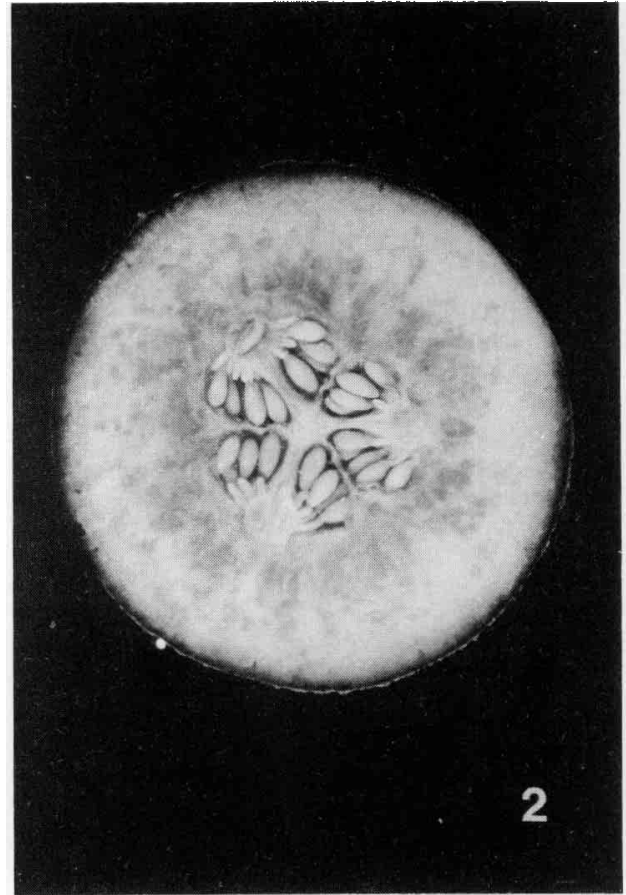

Figure 2

Marbrure interne des fruits associée à l'isolat E15 de MYSV.

Internal mottle of the fruit associated with MYSV E15 isolate.
Témoin non inoculé.

Non inoculated control.

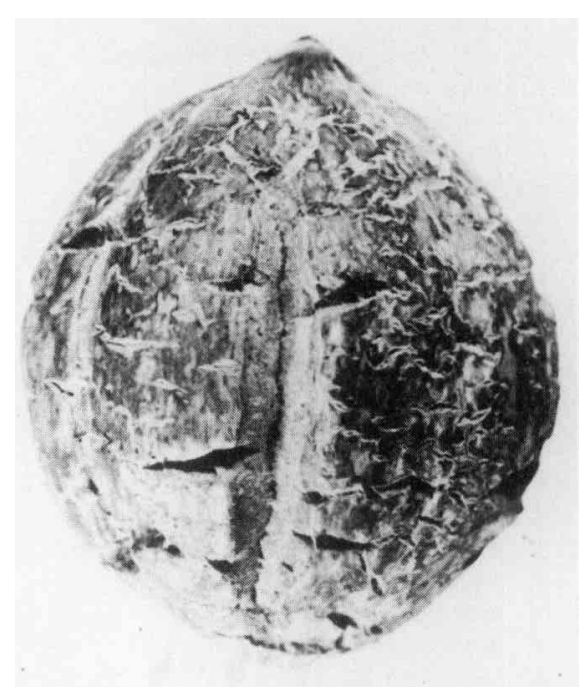

4
Figure 3a

Flétrissement et dessèchement de $\mathrm{C}$. melo var. "Doublon" (8 jours après l'inoculation) produit par l'isolat E9 de MYSV.

Wilting reaction induced in $\mathrm{C}$. melo var. "Doublon " by MYSV E9 isolate (8 days after inoculation).
Figure 3b

Témoin non inoculé.

Non inoculated control.
Figure 4

Craquelure externe des fruits associée à l'isolat E9 de MYSV.

External cracking of the fruit associated with MYSV E9 isolate. 
Freund. Les réactions sérologiques ont été réalisées en immunodiffusion en milieu gélosé en présence de dodécylsulfate de sodium (SDS) selon la méthode de PURCIFULL \& BATCHELOR (1977). Le milieu contient 0,8 p. 100 d'agar noble, 1 p. 100 d'azide de sodium et 0,5 p. 100 de SDS. Les puits utilisés ont $4 \mathrm{~mm}$ de diamètre et sont espacés entre eux de $4 \mathrm{~mm}$. Les antigènes bruts sont obtenus par filtration sur étamine du broyat de $1 \mathrm{~g}$ de feuilles dans $2 \mathrm{ml}$ d'eau distillée.

\section{RÉSULTATS}

\section{A. Gamme d'hôtes}

\section{Cucurbitacées}

Les isolats E9 (Sud-Est) et E15 (Sud-Ouest) provoquent des infections généralisées chez 28 des 34 espèces ou variétés de Cucurbitacées éprouvées (tabl. 1). Ils induisent chez la plupart d'entre elles des symptômes forts de rabougrissement et de mosaïque déformante.

\section{TABLEAU 1}

Gammes d'hôtes comparées des isolats E9 et E15 de MYSV et de l'isolat WMV2-FR

Comparative host ranges of $E 9$ and E15 isolates of MYSV and FR isolate of WMV2

$\overline{\text { Espèce (et variété) }}$

Isolat de virus

E9 E15 WMV2-FR

Cucurbitacées

Benincasa hispida (Thunb.) Cogn.

Bryonia cretica L. subsp. diö̈ca (Jacq.) Tutin

Citrullus lanatus (Thunb.) Mansfeld

Cucumis anguria $\mathrm{L}$.

C. dipsaceus Spach.

C. ficifolius A. Rich.

C. heptadactylus Naud.

C. melo L. var. « Doublon

C. melo L. var. "Védrantais"

C. melo L. var. 72025

C. melo L. var. B633

C. melo L. var. PI 161375

C. metuliferus Naud.

C. myriocarpus Naud.

C. sativus L. var. «Vert long maraîcher »

C. zeyheri Sond.

Cucurbita andreana Naud.

C. ecuadorensis Cutler \& Whitaker

C. ficifolia Bouché

C. foetidissima H.B.K

C. gracilior Bailey

C. lundelliana Bailey

C. martinezii Bailey

C. maxima Duch.

C. moschata Poir

C. okeechobeensis Bailey

C. pepo L. var. "Diamant»

C. sororia Bailey

Ecballium elaterium (L.) A. Rich.

Echinocystis wrightii (A. Gray) Cogn.

Lagenaria vulgaris Ser.

Luffa acutangula Roxb.

Momordica charantia $\mathrm{L}$.

Trichosanthes anguina $\mathrm{L}$

Amaranthacées

Gomphrena globosa L.

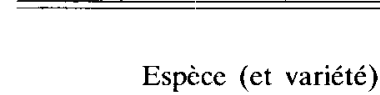

Isolat de virus

E9 E15 WMV2-FR

Apocynacées

Vinca rosea $\mathrm{L}$.

Caryophyllacées

Stellaria media (L.) Vill.

Chenopodiacées

Beta vulgaris $\mathrm{L}$.

Chenopodium amaranticolor Coste et

Reyn.

C. quinoa Willd

Spinacia oleracea L.

Composées

Cichorium endivia $\mathrm{L}$.

Lactuca sativa $\mathrm{L}$.

Senecio vulgaris $\mathrm{L}$.

Zinnia elegans Jacq.

Crucifères

Brassica oleracea L. var. botrytis

Brassica napus (L.) Metzger

Capsella bursapastoris (L.) Medik

Labiées

Lamium amplexicaule $\mathrm{L}$.

Ocimum basilicum L.

Légumineuses

Phaseolus aureus Roxb.

P. vulgaris L. var. GN 1140

$P$. vulgaris L. var. «Dark Red Kidney»

$P$. vulgaris L. var. "Pinto"

$P$. vulgaris L. var. PI 109958

P. vulgaris L. var. PI 203859

Pisum sativum L. var. "Juwel"

$P$. sativum L. var. "Alaska»

P. sativum L. var. "Diamant"

Trifolium repens $\mathrm{L}$.

Vicia faba $\mathbf{L}$.

Vigna sinensis (Endl.) L.

Malvacées

Lavatera trimestris $\mathrm{L}$.

Ombellifères

Apium graveolens $\mathrm{L}$.

Portulaccacées

Portulacca oleracea L.

Renonculacées

Ranunculus sardous Crantz

Scrophulariacées

Torenia fournieri Lind.

Solanacées

Capsicum annuum L. var. "Yolo Wonder"

Datura stramonium L.

Lycopersicon esculentum Mill. var. «Monalbo"

Nicotiana clevlandii A. Gray

$N$. glutinosa $\mathbf{L}$

N. tabacum L. var. "Paraguay"

N. tabacum L. var. "Samsun »

N. tabacum L. var. « Xanthi»

Petunia hybrida Vilm.

Solanum melongena $\mathrm{L}$.

Valerianacées

Valerianella olitoria Poll.

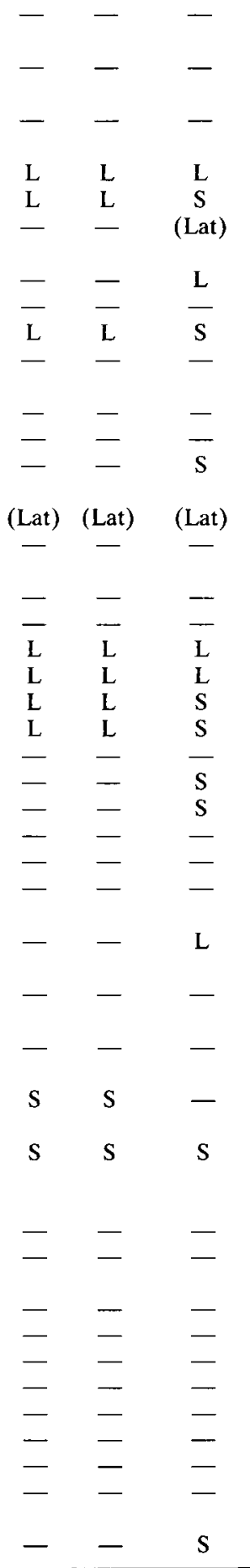

- : absence de symptômes; le virus n'cst pas retrouvé à l'apex des plantes

$\mathrm{L}$ : lésions locales sur les organes inoculés et absence de symptômes généralisés ; le virus n'est pas retrouvé à l'apex des plantes

$\mathrm{S}$ : présence de symptômes généralisés ; le virus est retrouvé à l'apex des plantes

(Lat) : absence de symptômes; le virus est cependant présent à l'apex des plantes.

- : no symptom; the virus is not recovered from the plant apex L: local lesions but no systemic symptom; the virus is not recovered from the plant apex

$S$ : systemic symptoms ; the virus may be recovered from the plant apex

(Lat) : no symptom ; the virus may however be recovered from the plant apex 
Chez C. melo var. «Védrantais », les isolats E9 et E15 induisent les mêmes symptômes: les plantes inoculées présentent d'abord un éclaircissement des nervures, suivi d'un jaunissement général accompagné parfois de l'apparition de taches ou cloques vert foncé localisées (fig. 1a). La croissance est alors sévèrement ralentic et la plante reste naine. Plus tard un ou plusieurs axes végétatifs peuvent se développer en donnant naissance à des feuilles très déformées présentant souvent des énations.

Chez C. melo var. «Doublon », l'isolat E9 induit, 7 à $10 \mathrm{j}$ après l'inoculation, le flétrissement de l'apex de la plante suivi d'une nécrose généralisée et de la mort (fig. 3a). L'isolat E15 induit chez cette variété des symptômes très voisins de ceux qu'il provoque chez la variété «Védrantais ».

Les symptômes sur fruits associés à ces virus, craquelure de l'écorce et marbrure de la chair (fig. 2 et 4), ont été reproduits en serre chez des plantes inoculées mécaniquement. Cependant, en particulier pour l'isolat E15, les symptômes internes semblent beaucoup plus fréquents lorsque le virus est apporté en mélange avec le CMV, incapable par lui-même d'induire de tels symptômes.

Une infection par le virus E15 provoque chez le melon une réduction importante du poids moyen des graines et de leur pouvoir germinatif. Ainsi un poids moyen de 50 graines de $378 \mathrm{mg}$ et un taux de germination de 20,8 p. 100 ont été établis pour des graines récoltées sur des plantes infectées par l'isolat E15 alors qu'ils étaient respectivement de $1498 \mathrm{mg}$ et de 92,8 p. 100 (différences hautement significatives) pour des graines récoltées sur des plantes non inoculées.

Le WMV2-FR provoque chez ces 2 variétés des symptômes de «vein banding " accompagnés d'une déformation importante des feuilles. La croissance des tiges n'est pas sensiblement altérée.

Chez Cucurbita pepo L. var. "Diamant », les isolats E9 et E15 produisent d'abord des symptômes de jaunissement des feuilles qui présentent parfois quelques taches vert foncé ; après un arrêt passager de la croissance, les feuilles se développent en présentant un faciès filiforme (feuilles en lacets) et un «vein banding » prononcé. Chez les courgettes infectées par le WMV2-FR, les symptômes sont plus faibles (mosaïque et « vein banding» légers).

Chez Cucumis sativus L. var. «Vert long maraîcher », les isolats E9 et E15 provoquent une mosaique forte, accompagnée de déformations foliaires prononcées et d'une réduction de croissance. Le WMV2-FR, par contre, ne produit pas de symptômes chez cette variété, bien qu'il s'y multiplie de façon systémique.

Chez Luffa acutangula Roxb., les isolats E9 et E15 provoquent des symptômes très forts (déformations foliaires, mosaique), contrairement au WMV2-FR qui n'infecte pas cette espèce. Les rétroinoculations à $C$. melo var. «Doublon» par la voie mécanique donnant des résultats irréguliers, la présence ou l'absence de virus à l'apex des plantes de $L$. acutangula a été établie par des tests sérologiques et des rétroinoculations par pucerons.

\section{Autres familles botaniques}

Les isolats E9 et E15 provoquent des lésions locales chez 8 des espèces ou variétés éprouvées sans toutefois se généraliser chez ces hôtes (tabl. 1). Seules 3 espèces ont été infectées de façon systémique : Lamium amplexicaule L., Ranunculus sardous Crantz. et Torenia fournieri Lind., les symptômes produits chez ces hôtes sont faibles ou nuls.

Le WMV2-FR provoque des lésions locales chez les mêmes hôtes que les isolats E9 et E15 (mais se généralise chez 4 d'entre eux) ainsi que chez Cichorium endivia L. et Lavatera trimestris L.. Il infecte de façon systémique 10 des espèces ou variétés envisagées.

\section{B. Transmission par pucerons}

Les isolats E9 et E15 sont très efficacement transmis par Myzus persicae Sulz. et Aphis gossypii Glov. sur le mode non persistant (tabl. 2).

\section{TABLEAU 2}

Taux de transmission des isolats E9 et E15 de MYSV exprimés en p. 100 de plantes (C. melo var. "Doublon") infectées (40 ou 50 plantes ont été inoculées par 3 pucerons viruliferes pour les isolats E9 et E15 respectivement)

Transmission rates established for E9 and E15 isolates of MYSV expressed as a percentage of infected plants $(40$ or 50 plants (C. melo var. "Doublon") were inoculated with 3 viruliferous aphids for E9 and E15 isolates respectively)

\begin{tabular}{|c|c|c|}
\hline \multirow[b]{2}{*}{ Puceron } & \multicolumn{2}{|c|}{ Isolat } \\
\hline & E9 & E15 \\
\hline Myzus persicae & 72 & 84 \\
\hline Aphis gossypii & 92 & 72 \\
\hline
\end{tabular}

\section{Microscopie électronique}

Les isolats E9 et E15 apparaissent, en coloration négative, sous forme de filaments flexueux (fig. 5). Ils sont souvent associés, en «dip method», à des structures paracristallines semblables à celles observées avec de nombreux potyvirus (EDWARDSON, 1974). La mensuration de

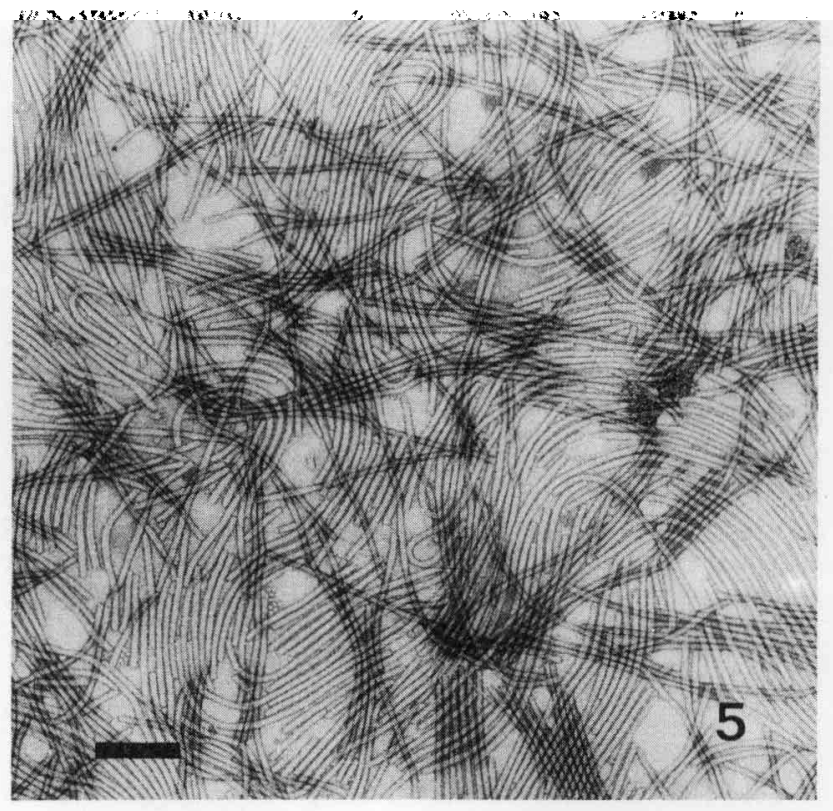

Figure 5

Particules virales purifiées de l'isolat E15 de MYSV (1a barre représente $200 \mathrm{~nm}$ ).

Purified virus particles of MYSV E15 isolate (bar represents $200 \mathrm{~nm})$. 
70 particules a permis d'établir une longueur moyenne de $735 \mathrm{~nm}$ pour l'isolat E9 et celle de 160 particules, une longueur moyenne de $725 \mathrm{~nm}$ pour l'isolat E15.

\section{Sérologie}

Aucune réaction sérologique n'est observée lorsqu'on confronte les isolats E9 et E15 en immunodiffusion SDS à des antisérums préparés contre les virus suivants : virus de la mosaïque de la pastèque type 1 (WMV1) (sérums fournis par H. LOT \& D. PuRCIFUlL), virus de la mosaique de la pastèque type «Maroc» (WMV-MOR) (sérum fourni par D. Purcifull), virus de la mosaique jaune du haricot (BYMV) (sérum préparé par V. LiSA et fourni par O. LOVISOLO). Réciproquement ces différents virus n'ont pas réagi avec un antisérum préparé contre l'isolat E15.

Le comportement des isolats E9 et E15 vis-à-vis d'antisérums préparés contre le virus de la mosaíque de la pastèque type 2 (WMV2) varic selon le titre de l'antisérum (tabl. 3).

\section{TABLEAU 3}

Titres établis en immunodiffusion SDS vis-à-vis de WMV2-FR et des isolats E9 et E15 de MYSV pour divers antisérums.

Titers established for various antiserums towards WMV2-FR and E9 and E15 MYSV isolates in SDS immunodiffusion test.

\begin{tabular}{llccc}
\hline \hline \multirow{2}{*}{ antisérum (a) } & \multicolumn{4}{c}{ Antigène } \\
& WMV2-FR & E9 & E15 & $\begin{array}{c}\text { Extraits de } \\
\text { melon sain }\end{array}$ \\
& & & & \\
\hline WMV2-USA & $1 / 256$ & $1 / 16$ & $1 / 16$ & 0 \\
WMV2-FR & $1 / 16$ & $0-1 / 1$ & $0-1 / 1$ & 0 \\
WMV2-N.I. & $1 / 8$ & 0 & 0 & 0 \\
E15 & $0-1 / 1$ & $1 / 2$ & $1 / 2$ & 0 \\
\hline
\end{tabular}

(a) Les antisćrums WMV2-USA et WMV2-FR nous ont été fournis par D. PuRCIFULL et H. LoT. L'antisérum WMV2-N.I. (North Italy) nous a été fourni par V. LisA et O. LovisOLo.

(a) WMV2-USA and WMV2-FR antiserums were kindly supplicd by D. PURCIIULL and H. LOT. WMV2-N.I. (North Italy) antiserum was kindly supplied by $V$. LISA and O. LOVISOLO.

Lorsque celui-ci est suffisamment élevé, les isolats E9 et E15 produisent chacun un arc de précipitation; ceux-ci se raccordent parfaitement entre eux, et forment un éperon avec l'arc de précipitation du WMV2 (fig. 6). Les isolats E9 et E15 réagissent avec l'antisérum préparé contre l'isolat E15 en formant également chacun un arc de précipitation. Ces arcs se rejoignent parfaitement lorsque les isolats E9 et E15 sont confrontés côte à côte (fig. 6). Un arc léger apparaît parfois lorsqu'on éprouve le WMV2-FR vis-à-vis de l'antisérum E15. L'irrégularité de cette réaction peut être attribuée à la faiblesse du titre de l'antisérum (tabl. 3). Aucun de ces sérums n'a réagi avec des extraits de melons sains.

Ces résultats suggèrent d'une pait l'existence d'une étroite parenté sérologique entre les isolats E9 et E15 (leur identité sérologique ne pouvant être établie qu'en les éprouvant également vis-à-vis d'un antisérum E9) et, d'autre part, l'existence d'une parenté sérologique éloignćc entre ces isolats et le WMV2.

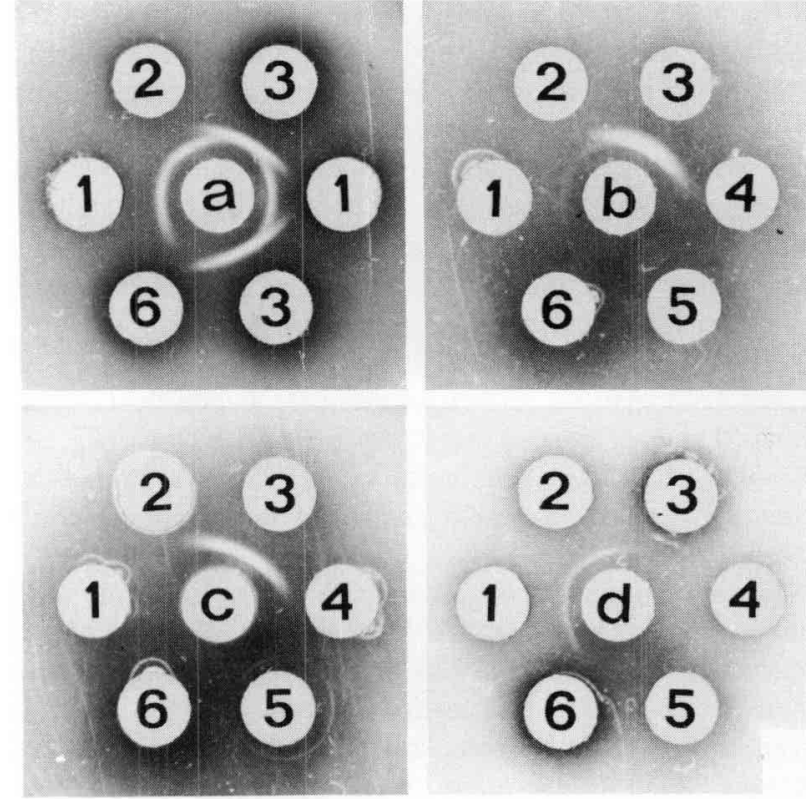

Figure 6

Réactions sérologiques en immunodiffusion SDS (1-4) extraits bruts de melons infectés par (1) l'isolat E9 de MYSV (2) l'isolat E15 de MYSV (3) l'isolat WMV2-FR (4) un isolat français de WMV1 (5) extraits de C. pepo infecté par le WMV-MOR dans 1,5 p. 100 SDS (6) extraits de melon sain; (a) antisérum WMV2-USA, (b) antisérum WMV2-FR (c) antisérum WMV2-N.I., (d) antisérum MYSV isolat E15, (5) et (a) ont été fournis par D. PURCIFULL, (b) par H. Lot et (c) par V. LISA et O. Lovisolo.

Serological reactions in $S D S$ immunodiffusion (1-4) Muskmelon extracts from plants infected by (1) MYSV E9 isolate (2) MYSV E15 isolate (3) WMV2-FR isolate (4) French WMV1 isolate (5) SDS extract of $\mathrm{C}$. pepo infected by WMV-MOR (6) healthy muskmelon extract (a) WM2-USA antiserum (b) WMV2-FR antiserum (c) WMV2-N.I. antiserum (d) MYSV E15 antiserum. (5) and (a) were kindly supplied by D. PURCIFULL, (b) by H. LOT and (c) by $V$. LISA and O. LOVISOLO.

\section{E. Transmission par la graine}

Les graines de 34 fruits récoltés sur des plantes infectées par l'isolat E15 (seul ou en mélange avec le CMV) ont été prélevées puis semées en terrines par groupes de 25 graines. Aucune des 1009 plantes obtenues (sur 2150 graines semées) ne présentait de symptômes viraux 2 mois après le semis.

\section{F. Essai de protection croisée}

Afin de mieux définir les liens de parenté existant entre les isolats E9, E15 et WMV2-FR, des essais de protection croisée ont été effectués en utilisant le marqueur intéressant qu'est le flétrissement de $C$. melo var. «Doublon » induit par l'isolat E9. Des groupes de $5 \mathrm{C}$. melo var. "Doublon" ont été inoculés soit par le WMV2-FR, soit par l'isolat E15 puis surinoculés à des temps variables par l'isolat E9.

Une infection par le WMV2-FR (tabl. 4) ne confère aucune protection vis-à-vis d'une surinoculation par l'isolat E9: en effet, dans tous les cas (sauf une plante sans doute échappée à l'inoculation), les plantes flétrissent. Par contre, une infection par l'isolat E15 confère une protection contre l'isolat E9, totale si celui-ci est apporté au moins $6 \mathrm{j}$ après la lère inoculation, partielle si elle intervient $3 \mathrm{j}$ seulement après celle-ci, nulle si les isolats E9 et E15 sont inoculés 
TABLEAU 4

Essai de protection croisée entre les isolats E15 et WMV2-FR d'une part et l'isolat E9 d'autre part

Cross protection test between E15 and WMV2-FR isolates, on one side and E9 isolate on the other side.

\begin{tabular}{cccl}
\hline \hline $\begin{array}{c}\text { Nature du } 1^{\text {er }} \\
\text { inoculum (a) }\end{array}$ & $\begin{array}{c}\text { Temps en jours entre } \\
\text { la } 1^{\text {re }} \text { et la } 2^{\mathrm{e}} \\
\text { inoculation }\end{array}$ & $\begin{array}{c}\text { Nature du } 2^{\mathrm{c}} \\
\text { inoculum }\end{array}$ & Symptomes(c) \\
\hline- (b) & - & - & $5 / 5 \mathrm{O}$ \\
WMV2-FR & - & - & $5 / 5 \mathrm{VB}$ \\
E15 & - & - & $5 / 5 \mathrm{JR}$ \\
E9 & - & - & $5 / 5 \mathrm{~F}$ \\
WMV2-FR+E9 & - & - & $5 / 5 \mathrm{~F}$ \\
E15 + E9 & - & - & $5 / 5 \mathrm{~F}$ \\
WMV2-FR & 3 & E9 & $5 / 5 \mathrm{~F}$ \\
E15 & 3 & E9 & $3 / 5 \mathrm{~F} 2 / 5 \mathrm{JR}$ \\
- & - & E9 & $5 / 5 \mathrm{~F}$ \\
WMV2-FR & 6 & E9 & $5 / 5 \mathrm{~F}$ \\
E15 & 6 & E9 & $5 / 5 \mathrm{JR}$ \\
- & - & E9 & $5 / 5 \mathrm{~F}$ \\
WMV2-FR & 10 & E9 & $5 / 5 \mathrm{~F}$ \\
E15 & 10 & E9 & $5 / 5 \mathrm{JR}$ \\
- & - & E9 & $5 / 5 \mathrm{~F}$ \\
WMV2-FR & 14 & E9 & $4 / 5 \mathrm{~F} 1 / 5 \mathrm{VB}$ \\
E15 & 14 & E9 & $5 / 5 \mathrm{JR}$ \\
- & - & E9 & $5 / 5 \mathrm{~F}$ \\
WMV2-FR & 21 & E9 & $5 / 5 \mathrm{~F}$ \\
E15 & 21 & $5 / 5 \mathrm{JR}$ \\
- & - & $5 / 5 \mathrm{~F}$ \\
& & &
\end{tabular}

(a) 5 plantes de $C$. melo var. «Doublon» ont été utilisées par est.

(b) - indique qu'il n'y a pas eu d'inoculation.

(c) exprimés en nombre de plantes présentant un symptôme divisé par le nombre total de plantes inoculées:

$\mathrm{O}$ : pas de symptômes

VB : « vein banding " (symptôme associé au WMV2-FR)

JR: Jaunissement et rabougrissement (symptôme associé à l'isolat E15)

F : Flétrissement (symptôme associé à l'isolat E9)

(a) Five C. melo var. "Doublon" were used per test

(b) - means that no inoculum was applied

(c) expressed as number of plants showing symptom divided by the total number of plants inoculated

$\mathrm{O}:$ no symptom

VB : Vein banding (WMV2-FR symptom)

JR: Yellow Stunt (E15 isolate symptom)

$\mathrm{F}$ : Wilt (E9 isolate symptom)

simultanément. Des rétroinoculations à $2 \mathrm{C}$. melo var. «Doublon » d'extraits de plantes inoculées par l'isolat E15 puis par l'isolat E9 et ne flétrissant pas ont conduit à l'apparition de symptômes de jaunissement et de rabougrissement ; ceci confirme que l'isolat E9 ne s'était pas multiplié chez ces plantes. Ces résultats suggèrent une étroite parenté entre les isolats E9 et E15 qui ne semble pas exister entre les isolats E9 et WMV2-FR.

\section{DISCUSSION ET CONCLUSIONS}

Les isolats E9 et E15 présentent des gammes d'hôtes identiques et induisent chez une même espèce, des symptômes très voisins, à l'exception de la réaction de certaines variétés de melon telles que «Doublon ». Les rétroinoculations, réalisées systématiquement à partir des diverses plantes envisagées dans l'étude de la gamme d'hôtes, ont toujours permis de retrouver sur le melon «Doublon» le faciès maladif associé au virus inoculé. Lors des essais de transmission des isolats E9 et E15 par aphides, les melons «Doublon» inoculés ont également toujours montré les symptômes caractéristiques du virus étudié. Ces observations ainsi que les résultats des essais de protection croisée (une inoculation par l'isolat E15 protège contre l'isolat E9) semblent exclure l'hypothèse selon laquelle l'isolat E9 représenterait un mélange de virus comprenant l'isolat E15 et un autre composant viral qui serait responsable du flétrissement observé chez la variété «Doublon». Il apparaît donc que les isolats E9 et E15, qui présentent en outre des caractéristiques morphologiques, sérologiques et des vecteurs communs, sont des souches d'un même virus différant entre elles seulement par leurs aptitudes pathogènes à l'égard de certaines variétés de melon.

Plusieurs propriétés permettent de rapprocher les isolats E9 et E15, analysés dans cette étude, du groupe des Potyvirus :

- l'aspect et la taille des particules virales : particules flexueuses d'environ $730 \mathrm{~nm}$;

- la présence dans les extraits bruts d'inclusions paracristallines souvent associées à ce type de virus (EDWARDSON, 1974) ;

- la transmission par les pucerons sur le mode non persistant ;

- la relation sérologique existant avec un membre de ce groupe (WMV2).

Cependant, une ou plusieurs propriétés définies pour les isolats E9 et E15 permettent de les différencier des Potyvirus déjà décrits et susceptibles d'infecter les Cucurbitacées.

Leur gamme d'hôtes relativement étendue à l'extérieur des Cucurbitacées, leur aptitude à infecter les variétés de melon B 633 et 72025 résistantes par hypersensibilité au WMV1 (WEBB \& SCOTT 1965, KAAN 1973) ainsi que l'absence de relation sérologique avec le WMV1 en font une entité différente de ce virus.

Leur aptitude à infecter L. acutangula immun au WMV2 (WEBB 1965, Russo et al. 1979), leur inaptitude à provoquer des lésions locales chez $L$. trimestris (MOLNAR \& SCHMELZER, 1964) ainsi qu'à infecter de façon systémique certaines variétés de Pisum sativum L. et Phaseolus vulgaris L. sensibles au WMV2 (SCHROEDER \& PROVVIDENTI, 1971 ; ProvVIDENTI, 1974) les font différer de ce virus. Ceci est confirmé par l'étude sérologique en immunodiffusion SDS et par les essais de protection croisée. Une analyse faisant intervenir d'autres méthodes sérologiques plus fines (telles que l'immunoélectromicroscopie) devrait cependant permettre de mieux définir les liens sérologiques existant entre ces virus.

La réaction de certains hôtes (tels que Chenopodium quinoa Willd., $L$. trimestris et $C$. melo var. «Védrantais») ainsi que les propriétés sérologiques permettent de différencier les isolats E9 et E15 du WMV type « Maroc » (FISCHER \& LOCKHART, 1974) lui-même non relié sérologiquement au WMV1 ni au WMV2 (PuRCIFUll \& HiEBERT, 1979 ; BAUM et al., 1979).

Enfin d'importantes différences de gammes d'hôtes séparent les isolats E9 et E15 du BYMV dont certains isolats infectent la courgette (PROVVIDENTI \& UYEMOTO, 1973) et du Bryonia Mottle Virus (LOCKHART \& FischeR, 1979).

L'ensemble de ces observations suggère donc que les isolats E9 et E15 appartiennent à une même entité virale non encore décrite, pour laquelle nous suggérons le nom de 
virus du rabougrissement jaune du melon ou de muskmelon yellow stunt virus (MYSV). Deux pathotypes de ce virus peuvent être distingués par l'aptitude à induire (pathotype F) ou non (pathotype NF) une réaction de flétrissement chez $C$. melo var. "Doublon".

Aucune des 165 variétés de melon éprouvées par RISSER et al. (1981) n'est résistante à l'isolat E9 de MYSV ; cependant, ces auteurs ont montré que la résistance à la transmission de virus par $A$. gossypii observée chez certaines variétés de melon (LECOQ et al., 1979 ; PITRAT \& LECOQ, 1980 ; LECOQ et al., 1980) est efficace vis-à-vis des isolats E9 et E15. Cette résistance peut contribuer à la protection des cultures de melon vis-à-vis du MYSV lorsque $A$. gossypii est un vecteur important au champ. Par ailleurs, la résistance de Cucurbita ecuadorensis Cutler \& Whitaker au MYSV (tabl. 1) est particulièrement intéressante car un programme de croisement interspécifique est en cours afin d'introduire chez $C$. pepo les résistances au $C M V$, au WMV1, au WMV2 et à l'oïdium présentes chez cette espèce sauvage (PITRAT \& DUMAS DE VAULX, 1979). Il est donc possible d'envisager la création de variétés résistantes au MYSV, chez la courgette.

Le nombre croissant des potyvirus capables d'infecter les Cucurbitacées pose le problème de leur identification dans des échantillons de plantes virosées. En effet, l'étude d'une gamme d'hôtes et l'observation d'extrait en microscopie électronique ne permettent pas toujours de lever certaines ambiguïés particulièrement dans le cas de mélanges de ces virus. L'association à ces méthodes d'une analyse sérologique en immunodiffusion en SDS devrait conduire à un diagnostic fiable et précis.

Reçu le 18 février 1981

Accepté le 16 juillet 1981

\section{REMERCIEMENTS}

Nous tenons à remercier Georgette RISSER pour avoir attiré notre attention sur le comportement particulier de certaines lignées de melon à l'isolat E9, G. Foullloux, H. LOT, V. LisA, O. Lovisolo \& D. Purcifull pour les graines ou les sérums qu'ils nous ont fournis, ainsi que $\mathrm{M}$. Duterl, Corinne AIllaud \& J. P. LERoux pour l'aide qu'ils nous ont apportée dans le cours de cette étude. Les photographies sont de J. RovGit:R.

Note: V. Lisa, G. Boccardo, G. D'agostino, G. DellaVALLE \& M. D'AQUILEIO (comm. pers.) ont récemment mis en évidence et caractérisé un potyvirus responsable d'une mosaïque jaune de la courgette. Ce virus, le Zucchini Yellow Mosaic Virus (ZYMV) présente une gamme d'hôtes proche du MYSV. Le ZYMV, n'est pas relié sćrologiquement au WMV1 ni au WMV2USA ; il présente, par contre, une lćgère parenté sérologique avec le WMV2-N.I. Ces affinités sérologiques semblent différencier le ZYMV du MYSV. Une étude comparative de ces 2 virus sera entreprise afin de mieux établir les relations pouvant exister entre eux.

\section{RÉFÉRENCES BIBLIOGRAPHIQUES}

Baum R. H., Purcifull D. E., Hiebert E., 1979. Purification and serology of a Moroccan isolate of Watermelon Mosaic Virus (WMV). Phytopathology, 69, 1021-1022.

Edwardson J. R., 1974. Some properties of the Potato Virus $Y$ Group. Fla. Agric. Exp. Stn Monograph Series, 398 p.

Fischer H. U., Lockhart B. E. L., 1974. Serious losses in Cucurbits caused by Watermelon Mosaic Virus in Morocco. Plant Dis. Rep. 58, 143-146.

Freitag J. H., Milne K. S., 1970. Host range, Aphid transmission and properties of Muskmelon Vein Necrosis Virus. Phytopatho$\log y, 60,166-170$.

Kaan F., 1973. Recherches sur la résistance du melon aux maladies notamment à la mosaïque de la pastèque et au mildiou, appliquées au type variétal «Cantaloup Charentais». C. R. Premières Journées d'études sur la sélection du melon. EUCARPIA, MontfavetAvignon (France) 19-22 Juin 1973, 41-49.

Lecoq H., Cohen S., Pitrat M., Labonne G., 1979. Resistance to cucumber mosaic virus transmission by aphids in Cucumis melo. Phytopathology, 69, 1223-1225.

Lecoq H., Labonne G., Pitrat M., 1980. Specificity of resistance to virus transmission by aphids in Cucumis melo. Ann. Phytopathol., 12, (2) 139-144.

Lockhart B. E. L., Fischer H., 1979. Host range and some properties of Bryonia Mottle Virus, a new member of the potyvirus group. Phytopathol. Z., 96, 244-250.

Luis Arteaga M. P., Quiot J. B., Leroux J. P., 1976. Mise en évidence d'une souche de Watermelon Mosaic Virus (WMV 2) dans le sud-est de la France. Ann. Phytopathol., 8, (3), 347-353.

Marrou J., 1967. Amélioration des méthodes de transmission mécanique des virus par absorption des inhibiteurs d'infection sur le charbon végétal. C. R. Acad. Agric. Fr., 53, 972-981.

Messiaen C. M., Maison P., Migliori A., 1963. Le Virus 1 du concombre dans le sud-est de la France. Phytopathol. méditerr., 2, 251-260.

Molnar A., Schmelzer K., 1964. Beiträge zur Kenntnis des Wassermelonenmosaik Virus. Phytopathol. Z., 51, 361-384.

Pitrat M., Dumas de Vaulx R., 1979. Recherche de géniteurs de résistance à l'oidium et aux virus de la mosaïque du concombre et de la mosaïque de la pastèque chez Cucurbita sp. Ann. Amélior. Plant., 29, 439-445.

Pitrat M., Lecoq H., 1980. Inheritance of resistance to Cucumber Mosaic Virus transmission by Aphis gossypii in Cucumis melo. Phytopathology, 70, 958-961.

Provvidenti R., 1974. Inheritance of resistance to Watermelon Mosaic Virus 2 in Phaseolus vulgaris. Phytopathology, 64, 14481450.

Provvidenti R., Uyemoto J. K., 1973. Chlorotic leaf spotting of Yellow Summer Squash caused by the severe strain of Bean Yellow Mosaic Virus. Plant Dis. Rep., 57, 280-282.

Purcifull D. E., Batchelor D. L., 1977. Immunodiffusion tests with Sodium dodecyl sulfate (SDS) treated plant viruses and plant viral inclusions. Fla. agric. Exp. Stn. tech. Bull. 788, 39 p.

Purcifull D. E., Hiebert E., 1979. Serological distinction of Watermelon Mosaic Virus isolates, Phytopathology, 69, 112-116.

Quiot J. B., Douine L., Gebre Selassie K., 1979. Fréquence des principales viroses identifiées dans une exploitation maraîchère du Sud-Est de la France. Ann. Phytopathol. 11, (3), 283-290.

Risser G., Pitrat M., Lecoq H., Rode J. C., 1981. Sensibilité variétale du melon (Cucumis melo L.) au virus du rabougrissement jaune du melon et à sa transmission par Aphis gossypii Glov. Hérédité de la réaction de flétrissement. Agronomie, 1, (10) 9-12.

Russo M., Martelli G. P., Vovlas C., Ragoxzino A., 1979. Comparative studies on mediterranean isolates of Watermelon Mosaic Virus. Phytopathol. mediterr., 18, 94-101.

Schroeder W. T., Provvidenti R., 1971. A common gene for resistance to Bean Yellow Mosaic Virus and Watermelon Mosaic Virus 2 in Pisum sativum. Phytopathology, 61, 846-848.

Spire D., Bertrandy J., Ferault C., 1966. Deux nouveaux virus de Cucurbitacées. Etudes de Virologie, Ann. Epiphyt., 17, ${ }^{\circ}$ H.S., 121-132.

Webb R. E., 1965. Luffa acutangula for separation and maintenance of Watermelon Mosaic Virus 1 free of Watermelon Mosaic Virus 2. Phytopathology, 55, 1379-1380.

Webb R. E., Scott H. A., 1965. Isolation and identification of Watermelon Mosaic Viruses 1 and 2. Phytopathology, 55, 895-900. 